\title{
Innate Immune Mechanisms in Myocardial Infarction - An Update
}

\author{
Razvan Gheorghita Mares ${ }^{1 *}$, Goran Marinkovic ${ }^{2}$, Ovidiu Simion Cotoi ${ }^{1}$, \\ Alexandru Schiopu ${ }^{1,2,3}$ \\ 1. University of Medicine and Pharmacy Targu Mures, Romania \\ 2. Experimental Cardiovascular Research Unit, Department of Clinical Sciences Malmö, \\ Lund University, Sweden \\ 3. Department of Cardiology, Skane University Hospital Malmö, Sweden
}

\begin{abstract}
Acute myocardial infarction (AMI) is a disease associated with high morbidity and mortality. Currently there are no available treatments specifically targeting the post-ischemic myocardial processes that lead to heart failure and recurrent coronary events. The innate immune system plays a central role in the two consecutive phases that follow an acute ischemic event: the inflammatory phase and the reparatory phase. The inflamatory phase involves a massive infiltration of neutrophils and inflammatory Ly6C hi monocytes into the injured myocardium. The reparatory phase is orchestrated by reparatory Ly6C ${ }^{l o}$ macrophages that clear necrotic and apoptotic cells through efferocytosis, secrete anti-inflammatory mediators and stimulate fibrosis and repair. Important recent studies provided proof that Ly6C hi monocytes that enter the myocardium in the inflammatory phase upregulate the orphan nuclear receptor Nr4al and switch phenotype to Ly6C $\mathrm{C}^{l o} \mathrm{Nr} 4 a 1^{\text {hi }}$ reparatory macrophages. Additionally, neutrophils have been shown to promote cardiac recovery by upregulating expression of the efferocytosis receptor MerTK on reparatory macrophages. A finely tuned balance between the inflammatory and the reparatory phases is thus essential for limiting myocardial damage and promoting efficient recovery. Treatment strategies targeting only the inflammatory phase have so far failed to improve prognosis in AMI patients. A detailed understanding of the interplay between the two phases of the innate immune response is paramount for designing efficient therapies able to improve postAMI prognosis. In the current review, we summarize the state-of-the-art of the field and discuss previous therapeutic attempts and currently ongoing clinical trials targeting innate immune mechanisms in AMI patients.
\end{abstract}

Keywords: myocardial infarction, innate immune system, monocytes/macrophages, neutrophils, myocardial repair

Received: 15 $5^{\text {th }}$ June 2017; Accepted: $28^{\text {th }}$ August 2017; Published: $15^{\text {th }}$ September 2017

*Corresponding author: Razvan Gheorghita Mares, University of Medicine and Pharmacy Targu Mures, 38 Gheorghe Marinescu Street, 540139, Targu Mures, Romania. E-mail: razvan_mares_7@yahoo.com 


\section{Introduction}

Acute myocardial infarction (AMI) is an acute ischemic event that occurs due to imbalance between oxygen supply and demand. Most commonly, AMI is caused by occlusion of a coronary artery due to rupture or erosion of an unstable atherosclerotic plaque, leading to sudden interruption of blood flow downstream of the occluded vessel (1). The ensuing myocardial ischemia and necrosis trigger a potent immune reaction involving two mechanistically distinct phases: the inflammatory phase and the reparatory phase. The early inflammatory response initiates digestion and removal of dead cardiac tissue, and paves the way for the reparative phase that involves resolution of inflammation, myofibroblast proliferation, scar formation and neovascularization (2). An optimal balance between the two phases is essential for adequate cardiac recovery. An exacerbated inflammatory phase amplifies the ischemic myocardial damage, leading to additional loss of systolic function. On the other hand, an extended reparatory phase might lead to excessive myocardial fibrosis and diastolic dysfunction (3).

Treatments targeting the innate immune response may provide important therapeutic strategies to reduce myocardial damage and to improve cardiac function and prognosis in AMI patients. However, previous immunosuppressive approaches have been deleterious in clinical studies, and a treatment able to adequately regulate the inflammation/repair balance has so far been elusive. In this review, we will address the present understanding of the cellular innate immune mechanisms active in the post-ischemic period, and discuss their impact on cardiac inflammation and repair. Emphasis will be placed on recent experimental evidence of paradigm-changing importance in this rapidly evolving field.

\section{Timeline of the innate immune response after myocardial infarction}

AMI triggers an abrupt rise in leukocyte numbers in blood and myocardium during the first hours and days. The two phases of the postAMI immune response involve different cellular populations and molecular processes. The initial inflammatory phase is defined by a robust influx of neutrophils into the myocardium, followed by Ly6C ${ }^{\text {hi }}$ inflammatory monocytes. The reparatory phase is characterized by an increased myocardial presence of Ly6 $\mathrm{C}^{\text {lo }}$ reparatory macrophages (4). Proper unfolding of the reparatory phase is conditioned by an efficient and well-balanced inflammatory phase. As discussed below, an excessive influx of neutrophils and inflammatory monocytes in the inflammatory phase leads to further myocardial damage and heart failure. The involvement of the innate immune cells in different stages of post-AMI pathology has attracted increasing attention in recent years.

\section{Days 1-3: Neutrophils}

Neutrophils are the first immune cell type to infiltrate the affected myocardium (5). Neutrophil infiltration peaks on day 1 post-AMI, and then gradually decreases (6). Necrotic cardiomyocytes and activated neutrophils release specific proteins that signal the presence of tissue injury, called danger-associated molecular patterns (DAMPs) or alarmins (7). DAMPs such as the high mobility group box-1 (HMGB1), S100 proteins or heat-shock proteins (HSPs) initiate the inflammatory response after $\mathrm{AMI}^{7}$. DAMPs bind to pattern recognition receptors (PRRs) on the surface of various cell types including neutrophils, monocytes/macrophages, and endothelial cells $(8,9)$. The most important PRRs involved in the pathogenesis of AMI are toll-like receptors 2 and 4 (TLR2 and TLR4), and the receptor for advanced glycation end-products (RAGE) (7). Binding of PRRs to these receptors acti- 
vates the transcription factor NF-kB, promoting inflammatory cell activation, increased expression of adhesion molecules, and secretion of a broad array of inflammatory mediators including alarmins, chemokines, and cytokines such as interleukin-1 $\beta$ (IL-1 $\beta$ ), IL-6, IL-8 and tumor necrosis factor- $\alpha$ (TNF- $\alpha)(10-13)$. These mediators promote endothelial activation and permeability, leading to further neutrophil recruitment in the infarcted area $(12,14,15)$. The role of neutrophils during the inflammatory phase is to initiate the clearance of necrotic cells and tissue debris, and to recruit monocytes into the infarcted myocardium. However, activated neutrophils also release high levels of reactive oxygen species (ROS) and proteolytic enzymes that promote further tissue damage during ischemia/ reperfusion (16). Additionally, experimental evidence suggests that neutrophils may induce death of viable cardiomyocytes by direct cytotoxic actions (16). In humans, studies have shown that high levels of neutrophils and their products are strongly related to the severity of coronary artery disease, size of ischemic injury and a deleterious prognosis in AMI patients (17).

Recent data by Ma et al. revealed for the first time that two different neutrophil sub-populations are present in the infarcted myocardium during the first 7 days post-AMI: the N1 and the N2 neutrophil subsets (6). N1 neutrophils are characterized by high expression of genes encoding the pro-inflammatory proteins $\mathrm{Ccl} 3$, $I l 1 \beta, I l 12 a$ and $T n f \alpha$, which are predominantly upregulated during the first 7 days after the AMI. N1 neutrophils are the most abundantly present neutrophils, representing $>80 \%$ of the total neutrophil population into the infarcted myocardium (6). In contrast, N2 neutrophils preferentially express the anti-inflammatory $\operatorname{Arg} 1, C d 206$, $I l 10$ and $\operatorname{Tg} f \beta$ genes, and their presence gradually increases during the first week post-AMI from around $2.5 \%$ on day 1 to $18 \%$ on day 7 (6).
The authors propose surface expression of the mannose receptor CD206, actively involved in phagocytosis, as a marker for distinguishing N2 neutrophils $\left(\mathrm{Ly}_{6 \mathrm{G}} \mathrm{CD}^{\mathrm{C}} 206^{+}\right.$) from their $\mathrm{N} 1$ counterparts (Ly6G $\left.{ }^{+} \mathrm{CD} 206^{-}\right)$. In-vitro treatment of neutrophils with cardiac cell lysate enriched in the HMGB1 and HSP60 alarmins predominantly polarized the cells towards a N1 phenotype. This effect was inhibited by a TLR4 blocking antibody, suggesting that TLR4 mediates alarmin-induced polarization of infiltrating neutrophils towards the pro-inflammatory subtype (6).

All the early data, both experimental and clinical, pointed towards a deleterious role of neutrophils in AMI. However, this paradigm has recently been changed by the findings of Horckmans et al., showing that neutrophil depletion using an anti-Ly6G antibody (clone 1A8) in a mouse model of AMI surprisingly led to defective myocardial recovery and progressive decline of cardiac function as assessed by echocardiography during the first 2 weeks postAMI (18). Neutrophil depletion led to defective efferocytosis due to decreased expression of the efferocytosis receptor myeloid-epithelial-reproductive receptor tyrosine kinase (MerTK) in anti-inflammatory macrophages infiltrating the infarcted myocardium. The inefficient efferocytosis was associated with excessive deposition of collagen fibers both in the infarcted area and in the remote myocardium (18). Thus, neutrophils are instrumental both in the inflammatory and in the reparatory phase by recruiting monocytes and shifting monocyte-to-macrophage polarization in the myocardium towards a reparatory MerTK $^{\text {hi }}$ phenotype.

\section{Days 3-5: Monocytes}

Two distinct monocyte populations have been described, both in mice and humans. In mice, monocyte phenotypic characterization is based on the expression of the chemokine receptors CCR2 and CX3CR1 (fraktalkine receptor, 
also known as G-protein coupled receptor 13 GPR13), the adhesion molecule CD62L (L-selectin), and the lymphocyte antigen 6C (Ly6C) (19). The classical or inflammatory monocyte subset expresses high levels of Ly6C, CCR2 and CD62L and low levels of CX3CR1. The CCR2 ${ }^{+}$ monocytes migrate towards the chemokine CCL2, also known as monocyte chemoattractant protein-1 (MCP-1), which is highly produced at inflammatory sites. The non-classical, or patrolling subset lacks Ly6C, CCR2, and CD62L but expresses high amounts of CX3CR1. The main chemoattractant for non-classical monocytes is fraktalkine (CXCL1) (20). Based on their function, the $\mathrm{Ly} 6 \mathrm{C}^{\mathrm{hi}} \mathrm{CCR} 2^{+} \mathrm{CD} 62 \mathrm{~L}^{+} \mathrm{CX} 3 \mathrm{CR} 1^{\text {lo }}$ mouse monocytes approximately correspond to human $\mathrm{CD} 14^{+} \mathrm{CD} 16^{-}$classical monocytes, whereas the Ly6C-CCR2-CD62L-CX3CR1 ${ }^{\text {hi }}$ cells correspond to non-classical or alternatively-activated $\mathrm{CD} 14^{+} \mathrm{CD} 16^{+}$human monocytes $(20)$. For convenience, in this review we will refer to these cells as Ly6C $\mathrm{C}^{\text {hi }}$ and $\mathrm{Ly} 6 \mathrm{C}^{\mathrm{lo}}$ monocytes.

Following neutrophils, monocytes are among the earliest responders that penetrate the injured myocardium (21). Importantly, neutrophils play a central role in inflammatory monocyte recruitment. It has recently been shown that this process is partly mediated by the neutrophil granular proteins cathelicidins (LL37 in humans; CRAMP in mice), which prime monocytes for endothelial adhesion by inducing conformational changes of beta1- and beta2-integrins on these cells (22). Several recent studies in both rodents and humans have investigated the dynamics of monocytes after AMI. Nahrendorf et al. demonstrated that ischemic injury in mice is followed by a sharp increase in the number of inflammatory Ly6 $\mathrm{C}^{\text {hi }}$ monocytes in the blood stream and in the heart, starting already during the first hours after the coronary occlusion and reaching peak numbers by days 3-4 (23). A large influx of monocytes in the inflammatory phase is thought to contribute to the initial cardiac injury, as CCR2 silencing using short interfering RNA significantly attenuated infarct size and improved heart functionality (24). The pro-inflammatory cytokine IL-1 $\beta$ was found to be one of the most important stimuli of post-ischemic myelopoesis, by stimulating hematopoietic stem-cell proliferation and by modulating the hematopoietic environment in the bone marrow (25). However, as the capacity of bone marrow to produce monocytes is insufficient to meet the large acute demand, an important part of monocyte production is outsourced to the spleen. The spleen has been shown to be the main initial monocyte provider in the first 24 hours after AMI (26), and also the main source of monocytes after this initial burst (27). Splenic myelopoesis is initiated by myeloid progenitor cells that migrate from the bone marrow under the influence of AMI-triggered sympathetic signals (28). Leuschner et al. found a robust expansion of the myeloid progenitor cells in the spleen post-AMI (27). IL-1 $\beta$ was present in high amounts in the spleen after an AMI, and was found to be a potent enhancer of extramedullary myelopoesis at this site (27). Importantly, splenectomy at the time of the AMI led to a significant drop in blood and cardiac monocytes, but led to impaired left ventricular ejection fraction, extended infarct size, thinner scar and accelerated left ventricular remodeling at 3 weeks after the ischemic event (27). These findings demonstrate that, similar to neutrophils, monocytes play important roles in cardiac repair and recovery.

In human studies, the post-AMI origin, kinetics and functions of monocytes have been shown to be very similar to those described in mice (29). It has recently been reported that human infarcted myocardium is sequentially infiltrated by 2 distinct monocyte subsets. Histopathological analysis of 40 hearts collected from patients who died at different time points after an AMI has showed that in the inflammatory phase (up to 5 days after $\mathrm{AMI}), 85 \%$ of the $\mathrm{CD} 14^{+}$monocytes in the 
infarct border zone were represented by the inflammatory $\mathrm{CD}^{-} 4^{+} \mathrm{CD} 16^{-}$subset, whereas an abundant presence of non-classical $\mathrm{CD} 14^{+} \mathrm{CD} 16^{+}$ monocytes was observed in the reparatory phase (5-14 days after AMI). Furthermore, the authors showed that monocyte recruitment after AMI in humans concurs with significantly decreased $\mathrm{CD}_{14}{ }^{+}$cells numbers in spleen in all phases of the AMI healing process ${ }^{(29)}$. Using cardiac MRI, Tsujioka et al. found that high circulating levels of inflammatory $\mathrm{CD} 14^{+} \mathrm{CD} 16^{-}$monocytes during the first week after the AMI correlated with low myocardial salvage 7 days after infarction, and with low left ventricular ejection fraction 6 months later. Moreover, cardiac MRI performed at 6 months after the AMI revealed that peak levels of the inflammatory $\mathrm{CD} 14^{+} \mathrm{CD} 16^{-}$subset correlated negatively with myocardial recovery (30). These data are well in line with the findings in rodents, confirming that excessive monocytosis in the immediate post-AMI inflammatory period is deleterious for long-term cardiac function and might represent a potential target for therapy in humans. However, any therapeutic intervention targeting monocyte numbers or function would have to be extremely finely-tuned in order not to impair their important roles in long-term cardiac repair and recovery.

\section{Days 5-7: Macrophages}

Under steady state conditions, tissue resident macrophages are mainly cells of embryonic origin with the ability of self-renewal (31). Recent fate mapping experiments revealed that most tissue-resident macrophage compartments are established prenatally and develop locally together with their respective host tissue (32). Maintaining the macrophage pool independently of the hematopoietic machinery requires self-renewal capacity $(33,34)$. However, under certain conditions including ischemia-induced inflammation, the cardiac macrophage pool is replenished by monocyte-derived cells (33).
During the first days following an ischemic injury, the majority of cardiac macrophages derive from inflammatory $\mathrm{Ly} 6 \mathrm{C}^{\mathrm{hi}}$ monocytes recruited from the blood pool. It was initially believed that there are two sequential waves of monocyte recruitment, followed by differentiation into two distinct macrophage populations (23). According to this hypothesis, the first phase involves recruitment of Ly6C $\mathrm{Ch}^{\text {hi }}$ monocytes, differentiating into classical M1 inflammatory macrophages that clear the cellular and matrix debris through efferocytosis. Subsequently, Ly$6 \mathrm{C}^{\text {lo }}$ monocytes were thought to differentiate into alternatively activated or reparatory M2 macrophages that promote resolution of inflammation and contribute to wound healing (23). Important recent experimental work using mice deficient in the transcription factor $\mathrm{Nr} 4 \mathrm{a} 1$ (nuclear receptor subfamily 4, group a, member 1) contradicts the sequential recruitment model and suggests that Ly6C ${ }^{\text {hi }}$ monocytes drive both the inflammatory and the reparative phase. $\mathrm{Nr} 4 \mathrm{a} 1$ is lacking in Ly6 $\mathrm{C}^{\text {hi }}$ monocytes, but is essential for the development of Ly6 $\mathrm{C}^{\mathrm{lo}}$ monocytes (35). Inflammatory Ly6C ${ }^{\text {hi }} \mathrm{Nr} 4 \mathrm{a} 1^{\text {lo }}$ monocytes massively infiltrate the myocardium during the early days after an AMI, gradually lose Ly6C surface antigen expression, upregulate $\mathrm{Nr} 4 \mathrm{a} 1$ and generate $\mathrm{Ly} 6 \mathrm{C}^{\mathrm{lo}} \mathrm{Nr} 4 \mathrm{a} 1^{\text {hi }}$ reparatory macrophages via local polarization (36). In Nr4a1 knockout mice, which do not have Ly6 $\mathrm{C}^{\text {lo }}$ monocytes, accumulation of Ly $6 \mathrm{C}^{\mathrm{lo}}$ macrophages in the infarcted myocardium is unaffected, providing strong evidence that these cells derive from Ly $6 \mathrm{C}^{\text {hi }}$ monocytes. However, in these mice the infiltrating $\mathrm{Ly} 6 \mathrm{C}^{\text {lo }} \mathrm{Nr} 4 \mathrm{a} 1^{-/-}$macrophages predominantly upregulate a set of pro-inflammatory genes, demonstrating that Nr4a1 has an important role in directing the infiltrating macrophages towards an anti-inflammatory and reparatory phenotype (36).

In the infarcted myocardium, macrophages are essential for the healing and regenerative processes in an efferocytosis-dependent manner. De- 
fective macrophage clearance of necrotic or apoptotic cells and damaged extracellular matrix leads to impaired collagen deposition and scar formation, causing adverse left ventricular remodeling and heart failure $(37,38)$. Novel insights into the post-AMI healing process indicate a critical role for the efferocytosis receptor MerTK, expressed by reparatory macrophages. Echocardiographic analysis at 7, 14 and 28 days after AMI in MerTK-deficient mice revealed impaired cardiac function and left ventricular remodeling, characterized by increased end-systolic and end-diastolic volumes compared to wild-type animals $(39,40)$. The absence of MerTK led to impaired efferocytosis of dying cardiomyocytes, but did not affect the number of inflammatory cells infiltrating the ischemic myocardium $(39,40)$. These effects were replicated in lethally irradiated wildtype mice transplanted with bone marrow isolated from MerTK ${ }^{-/}$animals. Conversely, transplantation of wild-type bone marrow carrying an intact Mertk gene into the MerTK ${ }^{-/-}$mice reversed the phenotype, suggesting that MerTK expression in bone marrow-derived myeloid cells is required and sufficient for an effective efferocytosis process (40). Similar results have been described for another phagocytosis receptor present on professional phagocytes, the milk fat globule epidermal growth factor 8 (Mfge8). Moreover, the effects of MerTK and Mfge8 were found to be slightly additive (39). Further support for the central importance of MerTK in cardiac repair was provided by mechanistic studies in neutrophil-depleted mice. Following AMI, the absence of neutrophils led to decreased MerTK expression in the reparatory macrophages, accumulation of apoptotic cells at the site of the infarction, worsened cardiac function, excessive fibrosis and heart failure ${ }^{18}$. These effects could be reversed by intraperitoneal administration of the protein neutrophil gelatinase-associated lipocalin (NGAL), present in the neutrophil secretome, indicating a crucial role of this protein in the neutrophil-macrophage crosstalk in-vivo (18).

\section{Fibrous scar formation}

The transition from Ly6 $\mathrm{C}^{\text {hi }}$ monocytes to Ly$6 \mathrm{C}^{\mathrm{lo}} \mathrm{MerTK} \mathrm{K}^{\mathrm{hi}}$ macrophages induces resolution of inflammation and promotes tissue repair. During the reparative phase, new extracellular matrix is synthesized to ensure mechanical stability and to preserve left ventricular structure ${ }^{41}$. If this process is impaired, it may lead to rupture of the weakened ventricular wall and sudden death. The Ly6C ${ }^{\text {lo }}$ macrophages support myocardium healing by secreting anti-inflammatory, pro-fibrotic and angiogenic mediators such as IL-10, transforming growth factor- $\beta 1$ (TGF- $\beta 1$ ) and vascular endothelial growth factor (VEGF) (2, 39). Myofibroblasts synthesize type I collagen that strengthens the infarcted area and prevents rupture. The origin of myofibroblasts in the infarcted myocardium remains an ongoing dispute, generated by experimental studies with contradictory results. Both bone marrow-derived fibroblasts and myofibroblasts (42), as well as a TGF- $\beta 1$-driven conversion of epicardial-derived cells (resident fibroblasts) into collagen-secreting myofibroblasts $(43,44)$ have been described. Importantly, at 5-7 days after AMI macrophages and endothelial cells orchestrate neovascularization in the border zone of the infarcted area, increasing blood supply to the remaining viable myocardium. This angiogenic response, regulated by members of the VEGF family, is crucial both to salvage ischemic myocardium "at risk" during the first week after the AMI, and to improve cardiac remodeling in the long term (45). Consequently, the formation of neovessels is a very important mechanism that limits infarct expansion and the transition to heart failure (45).

\section{The medium to long-term period after AMI}

As discussed above, a wealth of murine studies have mapped the innate immune events occurring during the first 2-3 weeks after AMI. Much less is known about the cellular and the molecular pathways involved in late cardiac re- 
modeling ultimately leading to end-stage heart failure. In both humans and animal AMI models it has been observed that chronic heart failure is characterized by persistently augmented levels of pro-inflammatory mediators (46). Elevated levels of several inflammatory cytokines and chemokines including TNF- $\alpha$, IL- $1 \beta$, IL-6, MCP-1 correlate with heart failure severity and with a poor prognosis (47).

A recent study by Ismahil et al. indicates that chronic post-ischemic heart failure in mice is accompanied by elevated numbers of pro-inflammatory monocytes, macrophages and dendritic cells in blood and in the heart (48). The authors suggest that the spleen plays a crucial role in the progression of cardiac remodeling, by persistent mobilization of inflammatory monocytes promoting an ongoing low-grade inflammatory response in the myocardium. In mice with established post-ischemic chronic heart failure, splenectomy performed at 8 weeks after the AMI reversed cardiac remodeling, improved left ventricle ejection fraction and attenuated macrophage infiltration into the heart. In contrast, mice undergoing sham abdominal surgery without splenectomy continued to exhibit progressive cardiac remodeling and functional decline (48). Moreover, adaptive transfer of mononuclear splenocytes isolated from mice with heart failure to naive mice led to left ventricle dilation, systolic dysfunction and myocardial fibrosis (48). AMI patients frequently have several cardiovascular risk factors and associated comorbidities associated with chronic low-grade inflammation such as diabetes (49), obesity (50) or renal failure. These factors might lead to an exaggerated and prolonged inflammatory response after AMI, delayed reparatory mechanisms and more severe heart failure. Further studies on leukocyte activity and their role in late cardiac remodeling might allow us to identify potential therapeutic targets to prevent progressive development of post-AMI heart failure.
Despite modern reperfusion therapy with percutaneous coronary intervention and stenting, as well as rigorous control of cardiovascular risk factors, the short- and long-term incidence of recurrent coronary events is still high (51). The mechanisms responsible for the heightened susceptibility for re-infarction remain to be characterized, but a chronic activation of innate immune mechanisms is thought to be highly relevant in this context (52). In mice with already developed atherosclerosis, Dutta et al. have shown that the AMI-triggered inflammatory process increases the size and inflammatory cell content of atherosclerotic plaques (28). Protease activity was imaged in aortic atherosclerotic plaques by hybrid fluorescence molecular tomography-X-ray computed tomography (FMT-CT), before and 3 weeks after the AMI. The results showed a potent increase of plaque protease activity in parallel with an increased number of inflammatory cells in the atherosclerotic plaque core, particularly the Ly6C ${ }^{\text {hi }}$ monocyte subset. The spleen has been identified as the most important provider of inflammatory monocytes, as atherosclerosis did not increase in mice that have been splenectomized at the time of the AMI. Furthermore, the authors demonstrated that under the influence of heightened sympathetic nervous activity after AMI, progenitor stem cells egress from the bone marrow, seed the spleen and amplify extramedullary myelopoiesis and monocyte deployment to atherosclerotic sites (28). Consequently, leukocyte accumulation within the plaques amplify plaque vulnerability, increasing the risk for rupture and thrombosis.

\section{Anti-inflammatory therapeutic approaches in AMI}

Early studies suggesting that inflammation is deleterious and has a significant impact on longterm prognosis have prompted interest in developing effective anti-inflammatory strategies in 
AMI patients. However, potent anti-inflammatory treatment with glucocorticoids was associated with impaired myocardial healing, development of ventricular aneurysms and high risk of ventricular rupture in clinical studies $(53,54)$. Similar detrimental effects were observed following treatment with non-steroidal anti-inflammatory drugs (55). In consequence, glucocorticoids and non-steroidal anti-inflammatory drugs are contraindicated in patients with AMI by the current clinical guidelines (56). The failure of these trials revealed the need for more specific and finely-tuned immunomodulatory approaches in AMI.

Blockade of the pro-inflammatory cytokine IL-1 $\beta$ has emerged in recent years as a potential therapeutic approach to reduce cardiovascular risk in AMI survivors. In apolipoprotein E-defficient $\left(\mathrm{ApoE}^{--}\right)$mice with induced AMI, antibody-mediated IL-1 $\beta$ signaling blockade dampened the exacerbated myelopoesis triggered by the ischemic injury, leading to a lower degree of myocardial inflammation and improved cardiac function at 3 weeks after the infarction (25). Recently, the MRC-ILA Heart study, a phase II double-blinded randomized placebo-controlled trial tested the effects of the recombinant IL- $1 \beta$ receptor antagonist Anakinra on systemic inflammation and prognosis in 182 non-ST-elevation acute coronary syndrome (NSTE-ACS) survivors (57). The treatment, administered in the acute 14-day period immediately after the AMI, significantly reduced the levels of the inflammatory biomarker C-reactive protein (CRP) at 7 and 14 days compared to placebo (57). However, the incidence of major adverse cardiovascular events (MACE) at 1-year was higher in the active treatment group, suggesting that IL-1 $\beta$ blockade should not be used clinically in the immediate post-AMI period. In contrast, the large ongoing CANTOS trial will evaluate the effects of IL-1 $\beta$ blockade with the recombinant human monoclonal antibody Canakinumab in stable
post-AMI patients with persistently elevated level of systemic inflammation after the acute period. This intention-to-treat study will include a total of 17200 AMI survivors randomized to receive 50,150 or $300 \mathrm{mg}$ antibody or placebo subcutaneously every 3 months for the duration of the trial (approximately 4 years). Only patients with persistent elevation of high sensitivity C-reactive protein $>2 \mathrm{mg} / \mathrm{L}$ post-AMI despite application of secondary-prevention strategies will be included, as these patients are considered to have a high residual cardiovascular risk (58). The primary end-point will be a composite of recurrent AMI, stroke, and cardiovascular death.

\section{Concluding summary and future directions}

In summary, important recent discoveries have shed light on the systemic and local innate immune mechanisms triggered by myocardial ischemia. The first events following myocardial ischemia are the release of alarmins from dead cardiomyocytes and the activation of the sympathetic nerve system. Subsequently, pro-inflammatory myeloid cells are released from the bone marrow and spleen and infiltrate the myocardium. Elevated levels of IL-1 $\beta$ in these hematopoietic compartments stimulate the accelerated rate of myelopoesis required to support the high leukocyte demand. Neutrophils are the first immune cells to infiltrate the myocardium, and initiate monocyte recruitment through endothelial activation, in a process partially mediated by cathelicidins (59). Recruitment of Ly6C ${ }^{\text {hi }}$ inflammatory monocytes is the cornerstone of myocardial recovery. These cells lose Ly6C expression, upregulate the transcription factor $\mathrm{Nr} 4 \mathrm{a} 1$ and are subsequently polarized into reparatory macrophages expressing the efferocytosis receptors MerTK and Mfge $8(36,39)$. Besides their roles in monocyte recruitment, neutrophils also secrete NGAL, which stimulates an efficient monocyte transition 
into reparatory macrophages (18). Finally, the reparatory macrophages clean necrotic cardiomyocytes and cellular debris through efferocytosis, gradually inhibit the inflammatory response, stimulate fibrosis and restore tissue homeostasis. The recognition of the efferocytosis receptors MerTK and Mfge8 as important contributors to the reparatory phase will undoubtedly lead to improved phonotypic characterization of reparatory macrophages and further discoveries in the field.

What has become increasingly clear is that a finely-tuned balance between the inflammatory and the reparatory phases is key for limiting myocardial damage and for efficient cardiac repair. As discussed above, all interventions that inhibit the initial inflammatory response invariably affect the reparatory phase as well, with deleterious long-term effects on cardiac function. Any potentially successful immunomodulatory therapy would have to limit the inflammatory damage to the myocardium, while improving or keeping intact the reparatory mechanisms. However, this has to be done with caution, as overly active myocardial repair mechanisms might lead to excessive fibrous tissue formation and diastolic heart failure. The results of the CANTOS trial are eagerly awaited, as this study will provide proof-of-concept whether inhibiting the inflammatory response in the post-acute period in AMI patients with persistently high systemic inflammation is beneficial. However, the largest amount of myocardial loss occurs in the first few hours and days after the ischemic injury, and the need for efficient immunomodulatory treatments to limit acute myocardial damage is currently not addressed. It is possible that targeted immunomodulatory therapies limited to this period might reduce the extent of the injury, while leaving the repair mechanisms unaffected. The recent findings reviewed here open new opportunities for intensified studies in this area, in order to promote efficient cardiac recovery, prevent heart failure and improve prognosis in AMI patients.

\section{Conflict of interest disclosure}

None of the authors has any conflict of interest to declare.

\section{Authors' contribution:}

RGM and GM performed the literature search and paper selection, and wrote the initial manuscript draft. OSC and AS designed the framework of the work, critically reviewed the manuscript for intellectual content and wrote the revised version. All authors have approved the final version of the manuscript and agree to be accountable for all aspects of the work.
Abbreviations:
AMI $=$ acute myocardial infarction
DAMPs $=$ danger-associated molecular patterns
HMGB1 = high mobility group box-1
PRRs $=$ pattern recognition receptors
$\mathrm{IL}=$ interleukin
$\mathrm{TNF}=$ tumor necrosis factor
ROS $=$ reactive oxygen species
TLR4 $=$ toll-like receptor 4
HSP60 $=$ heat shock protein 60
MerTK = myeloid-epithelial-reproductive re- ceptor tyrosine kinase
Ly6C = lymphocyte antigen 6C
MCP-1 = monocyte chemoattractant protein-1
$\mathrm{Nr} 4 \mathrm{a} 1$ = nuclear receptor subfamily 4 , group a, member 1
$\mathrm{TGF}=$ transforming growth factor
Mfge8 = milk fat globule epidermal growth fac- tor 8
NGAL $=$ neutrophil gelatinase-associated lipo- calin

\section{References}

1. Frangogiannis NG. Pathophysiology of Myocardial Infarction. Compr Physiol. 2015;5:1841-75. DOI: 10.1002/cphy.c150006

2. Prabhu SD, Frangogiannis NG. The Biological Basis for Cardiac Repair After Myocardial Infarction: From 
Inflammation to Fibrosis. Circ Res. 2016;119:91112. DOI: 10.1161/CIRCRESAHA.116.303577

3. Kain V, Prabhu SD, Halade GV. Inflammation revisited: inflammation versus resolution of inflammation following myocardial infarction. Basic Res Cardiol. 2014;109:444. DOI: 10.1007/s00395-014-0444-7

4. Swirski FK. Inflammation and repair in the ischaemic myocardium. Hamostaseologie. 2015;35:34-6. DOI: 10.5482/HAMO-14-09-0045

5. Yan X, Anzai A, Katsumata Y, Matsuhashi T, Ito K, Endo J, et al. Temporal dynamics of cardiac immune cell accumulation following acute myocardial infarction. J Mol Cell Cardiol. 2013;62:24-35. DOI: 10.1016/j.yjmcc.2013.04.023

6. Ma Y, Yabluchanskiy A, Iyer RP, Cannon PL, Flynn ER, Jung $M$, et al. Temporal neutrophil polarization following myocardial infarction. Cardiovasc Res. 2016;110:51-61. DOI: 10.1093/cvr/cvw024

7. Chan JK, Roth J, Oppenheim JJ, Tracey KJ, Vogl T, Feldmann M, et al. Alarmins: awaiting a clinical response. J Clin Invest. 2012;122:2711-9. DOI: 10.1172/ JCI62423

8. Arslan F, de Kleijn DP and Pasterkamp G. Innate immune signaling in cardiac ischemia. Nat Rev Cardiol. 2011;8:292-300. DOI: 10.1038/nrcardio.2011.38

9. Mann DL. The emerging role of innate immunity in the heart and vascular system: for whom the cell tolls. Circ Res. 2011;108:1133-45. DOI: 10.1161/CIRCRESAHA. 110.226936

10. Mathur S, Walley KR, Wang Y, Indrambarya T, Boyd JH. Extracellular heat shock protein 70 induces cardiomyocyte inflammation and contractile dysfunction via TLR2. Circ J. 2011;75:2445-52.DOI: 10.1253/circj.CJ11-0194

11. Riva M, Kallberg E, Bjork P, Hancz D, Vogl T, Roth J, et al. Induction of nuclear factor-kappaB responses by the S100A9 protein is Toll-like receptor-4-dependent. Immunology. 2012;137:172-82. DOI: 10.1111/j.13652567.2012.03619.x

12. Ryckman C, Vandal K, Rouleau P, Talbot M, Tessier PA. Proinflammatory activities of S100: proteins S100A8, S100A9, and S100A8/A9 induce neutrophil chemotaxis and adhesion. J Immunol. 2003;170:323342. DOI: 10.4049/jimmunol.170.6.3233

13. Scaffidi P, Misteli T, Bianchi ME. Release of chromatin protein HMGB1 by necrotic cells triggers inflammation. Nature. 2002;418:191-5. DOI: 10.1038/nature 00858

14. Kleinbongard P, Heusch G, Schulz R. TNFalpha in atherosclerosis, myocardial ischemia/reperfusion and heart failure. Pharmacol Ther. 2010;127:295-314. DOI: 10.1016/j.pharmthera.2010.05.002

15. Bujak M, Dobaczewski M, Chatila K, Mendoza LH, Li N, Reddy A, Frangogiannis NG. Interleukin-1 receptor type I signaling critically regulates infarct healing and cardiac remodeling. Am J Pathol. 2008;173:5767. DOI: 10.2353/ajpath.2008.070974

16. Vinten-Johansen J. Involvement of neutrophils in the pathogenesis of lethal myocardial reperfusion injury. Cardiovasc res. 2004;61:481-97. DOI: 10.1016/j.cardiores.2003.10.011

17. Carbone F, Nencioni A, Mach F, Vuilleumier N, Montecucco F. Pathophysiological role of neutrophils in acute myocardial infarction. Thromb Haemost. 2013;110:501-14. DOI: 10.1160/TH13-03-0211

18. Horckmans M, Ring L, Duchene J, Santovito D, Schloss MJ, Drechsler M, et al. Neutrophils orchestrate post-myocardial infarction healing by polarizing macrophages towards a reparative phenotype. Eur Heart J. 2017;38:187-197.

19. Geissmann F, Jung S, Littman DR. Blood monocytes consist of two principal subsets with distinct migratory properties. Immunity. 2003;19:71-82. DOI: 10.1016/ S1074-7613(03)00174-2

20. Gordon S, Taylor PR. Monocyte and macrophage heterogeneity. Nat Rev Immunol. 2005;5:953-64. DOI: $10.1038 /$ nri1733

21. Swirski FK, Nahrendorf M. Leukocyte behavior in atherosclerosis, myocardial infarction, and heart failure. Science. 2013;339:161-6. DOI: 10.1126/science. 1230719

22. Wantha S, Alard JE, Megens RT, van der Does AM, Doring Y, Drechsler M, et al. Neutrophil-derived cathelicidin promotes adhesion of classical monocytes. Circ Res. 2013;112:792-801. DOI: 10.1161/CIRCRESAHA. 112.300666

23. Nahrendorf M, Swirski FK, Aikawa E, Stangenberg L, Wurdinger T, Figueiredo JL, et al. The healing myocardium sequentially mobilizes two monocyte subsets with divergent and complementary functions. J Exp Med. 2007;204:3037-47.DOI: 10.1084/jem.20070885

24. Leuschner F, Dutta P, Gorbatov R, Novobrantseva TI, Donahoe JS, Courties G, et al. Therapeutic siRNA silencing in inflammatory monocytes in mice. Nat Biotechnol. 2011;29:1005-10. DOI: 10.1038/nbt.1989

25. Sager HB, Heidt T, Hulsmans M, Dutta P, Courties G, Sebas $\mathrm{M}$, et al. Targeting Interleukin-1beta Reduces Leukocyte Production After Acute Myocardial Infarction. Circulation. 2015;132:1880-90.

26. Swirski FK, Nahrendorf M, Etzrodt M, Wildgruber M, Cortez-Retamozo V, Panizzi P, et al. Identification of splenic reservoir monocytes and their deployment to inflammatory sites. Science. 2009;325:612-6. DOI: 10.1126/science. 1175202

27. Leuschner F, Rauch PJ, Ueno T, Gorbatov R, Marinelli B, Lee WW, et al. Rapid monocyte kinetics in acute myocardial infarction are sustained by extramedullary monocytopoiesis. J Exp Med. 2012;209:123-37. DOI: 10.1084/jem.20111009

28. Dutta P, Courties G, Wei Y, Leuschner F, Gorbatov 
R, Robbins CS, et al. Myocardial infarction accelerates atherosclerosis. Nature. 2012;487:325-9. DOI: 10.1038/nature 11260

29. van der Laan AM, Ter Horst EN, Delewi R, Begieneman MP, Krijnen PA, Hirsch A, et al. Monocyte subset accumulation in the human heart following acute myocardial infarction and the role of the spleen as monocyte reservoir. Eur Heart J. 2014;35:376-85. DOI: 10.1093/ eurheartj/eht331

30. Tsujioka H, Imanishi T, Ikejima H, Kuroi A, Takarada S, Tanimoto T, et al. Impact of heterogeneity of human peripheral blood monocyte subsets on myocardial salvage in patients with primary acute myocardial infarction. J Am Coll Cardiol. 2009;54:130-8. DOI: 10.1016/j.jacc.2009.04.021

31. Hashimoto D, Chow A, Noizat C, Teo P, Beasley $\mathrm{MB}$, Leboeuf $\mathrm{M}$, et al. Tissue-resident macrophages self-maintain locally throughout adult life with minimal contribution from circulating monocytes. Immunity. 2013;38:792-804. DOI: 10.1016/j.immuni.2013.04.004

32. Yona S, Kim KW, Wolf Y, Mildner A, Varol D, Breker $\mathrm{M}$, et al. Fate mapping reveals origins and dynamics of monocytes and tissue macrophages under homeostasis. Immunity. 2013;38:79-91. DOI: 10.1016/j.immuni.2012.12.001

33. Epelman S, Lavine KJ, Beaudin AE, Sojka DK, Carrero JA, Calderon B, et al. Embryonic and adult-derived resident cardiac macrophages are maintained through distinct mechanisms at steady state and during inflammation. Immunity. 2014;40:91-104. DOI: 10.1016/j. immuni.2013.11.019

34. Ginhoux F, Jung S. Monocytes and macrophages: developmental pathways and tissue homeostasis. Nat Rev Immunol. 2014;14:392-404. DOI: 10.1038/nri3671

35. Hanna RN, Carlin LM, Hubbeling HG, Nackiewicz D, Green AM, Punt JA, et al. The transcription factor NR4A1 (Nur77) controls bone marrow differentiation and the survival of Ly6C- monocytes. Nat Immunol. 2011;12:778-85. DOI: 10.1038/ni.2063

36. Hilgendorf I, Gerhardt L, Tan TC, Winter C, Holderried TA, Chousterman BG, et al. Ly-6Chigh Monocytes Depend on Nr4al to Balance both Inflammatory and Reparative Phases in the Infarcted Myocardium. Circ Res. 2014. DOI: 10.1161/CIRCRESAHA.114.303204

37. van Amerongen MJ, Harmsen MC, van Rooijen N, Petersen AH, van Luyn MJ. Macrophage depletion impairs wound healing and increases left ventricular remodeling after myocardial injury in mice. Am J Pathol. 2007;170:818-29. DOI: 10.2353/ajpath.2007.060547

38. Leblond AL, Klinkert K, Martin K, Turner EC, Kumar AH, Browne T, Caplice NM. Systemic and Cardiac Depletion of M2 Macrophage through CSF-1R Signaling Inhibition Alters Cardiac Function Post Myocardial Infarction. PLoS One. 2015;10:e0137515. DOI: 10.1371/ journal.pone.0137515
39. Howangyin KY, Zlatanova I, Pinto C, Ngkelo A, Cochain C, Rouanet M, et al. Myeloid-Epithelial-Reproductive Receptor Tyrosine Kinase and Milk Fat Globule Epidermal Growth Factor 8 Coordinately Improve Remodeling After Myocardial Infarction via Local Delivery of Vascular Endothelial Growth Factor. Circulation. 2016;133:826-39. DOI: 10.1161/CIRCULATIONAHA.115.020857

40. Wan E, Yeap XY, Dehn S, Terry R, Novak M, Zhang S, et al. Enhanced efferocytosis of apoptotic cardiomyocytes through myeloid-epithelial-reproductive tyrosine kinase links acute inflammation resolution to cardiac repair after infarction. Circ Res. 2013;113:1004-12. DOI: 10.1161/CIRCRESAHA.113.301198

41. Jugdutt BI. Ventricular remodeling after infarction and the extracellular collagen matrix: when is enough enough? Circulation. 2003;108:1395-403. DOI: 10.1161/01.CIR.0000085658.98621.49

42. Mollmann H, Nef HM, Kostin S, von Kalle C, Pilz I, Weber M, et al. Bone marrow-derived cells contribute to infarct remodelling. Cardiovasc res. 2006;71:66171. DOI: 10.1016/j.cardiores.2006.06.013

43. Ruiz-Villalba A, Simon AM, Pogontke C, Castillo MI, Abizanda G, Pelacho B, et al. Interacting resident epicardium-derived fibroblasts and recruited bone marrow cells form myocardial infarction scar. J Am Coll Cardiol. 2015;65:2057-66. DOI: 10.1016/j.jacc.2015.03.520

44. Yano T, Miura T, Ikeda Y, Matsuda E, Saito K, Miki $\mathrm{T}$, et al. Intracardiac fibroblasts, but not bone marrow derived cells, are the origin of myofibroblasts in myocardial infarct repair. Cardiovasc Pathol. 2005;14:2416. DOI: 10.1016/j.carpath.2005.05.004

45. Cochain C, Channon KM, Silvestre JS. Angiogenesis in the infarcted myocardium. Antioxidants \& redox signaling. 2013;18:1100-13. DOI: 10.1089/ars.2012.4849

46. Mann DL. Inflammatory mediators and the failing heart: past, present, and the foreseeable future. Circ Res. 2002;91:988-98. DOI: 10.1161/01. RES.0000043825.01705.1B

47. Bozkurt B, Mann DL, Deswal A. Biomarkers of inflammation in heart failure. Heart Fail Rev. 2010;15:33141. DOI: 10.1007/s10741-009-9140-3

48. Ismahil MA, Hamid T, Bansal SS, Patel B, Kingery JR, Prabhu SD. Remodeling of the mononuclear phagocyte network underlies chronic inflammation and disease progression in heart failure: critical importance of the cardiosplenic axis. Circ Res. 2014;114:266-82.DOI: 10.1161/CIRCRESAHA.113.301720

49. Odegaard JI, Chawla A. Pleiotropic actions of insulin resistance and inflammation in metabolic homeostasis. Science. 2013;339:172-7. DOI: $10.1126 /$ science. 1230721

50. Nagareddy PR, Kraakman M, Masters SL, Stirzaker RA, Gorman DJ, Grant RW, et al. Adipose tissue macrophages promote myelopoiesis and monocytosis in 
obesity. Cell metab. 2014;19:821-35. DOI: 10.1016/j. cmet.2014.03.029

51. Jernberg T, Hasvold P, Henriksson M, Hjelm H, Thuresson M, Janzon M. Cardiovascular risk in post-myocardial infarction patients: nationwide real world data demonstrate the importance of a long-term perspective. Eur Heart J. 2015;36:1163-70. DOI: 10.1093/eurheartj/ ehu505

52. Nahrendorf M, Swirski FK. Innate immune cells in ischaemic heart disease: does myocardial infarction beget myocardial infarction? Eur Heart J. 2016;37:86872. DOI: 10.1093/eurheartj/ehv453

53. Seropian IM, Toldo S, Van Tassell BW, Abbate A. Anti-inflammatory strategies for ventricular remodeling following ST-segment elevation acute myocardial infarction. J Am Coll Cardiol. 2014;63:1593-603. DOI: 10.1016/j.jacc.2014.01.014

54. Giugliano GR, Giugliano RP, Gibson CM, Kuntz RE. Meta-analysis of corticosteroid treatment in acute myocardial infarction. Am J Cardiol. 2003;91:1055-9. DOI: 10.1016/S0002-9149(03)00148-6

55. Gibson CM, Pride YB, Aylward PE, Col JJ, Goodman SG, Gulba D, et al. Association of non-steroidal anti-inflammatory drugs with outcomes in patients with ST-segment elevation myocardial infarction treated with fibrinolytic therapy: an ExTRACT-TIMI 25 analysis. J Thromb Thrombolysis. 2009;27:11-7. DOI: 10.1007/s11239-008-0264-4

56. Task Force on the management of ST-segment elevation acute myocardial infarction of the European Society of Cardiology (ESC), Steg PG, James SK, Atar D, Badano LP, Blomstrom-Lundqvist C, et al. ESC Guidelines for the management of acute myocardial infarction in patients presenting with ST-segment elevation. Eur Heart J. 2012;33:2569-619.

57. Morton AC, Rothman AM, Greenwood JP, Gunn J, Chase A, Clarke B, et al. The effect of interleukin-1 receptor antagonist therapy on markers of inflammation in non-ST elevation acute coronary syndromes: the MRC-ILA Heart Study. Eur Heart J. 2015;36:377-84. DOI: 10.1093/eurheartj/ehu272

58. Ridker PM, Thuren T, Zalewski A, Libby P. Interleukin-1beta inhibition and the prevention of recurrent cardiovascular events: rationale and design of the Canakinumab Anti-inflammatory Thrombosis Outcomes Study (CANTOS). Am Heart J. 2011;162:597-605. DOI: $10.1016 /$ j.ahj.2011.06.012

59. Swirski FK, Robbins CS. Neutrophils usher monocytes into sites of inflammation. Circ Res. 2013;112:7445. DOI: 10.1161/CIRCRESAHA.113.300867 